\title{
Direct Adsorption and Detection of Proteins, including Ferritin, onto Microlens Array (MA) Patterned Bioarrays
}

\author{
Feng Zhang, ${ }^{1}$ Richard J. Gates, ${ }^{1}$ Vincent S. Smentkowski, ${ }^{2}$ Sriram Natarajan, ${ }^{3}$ \\ Bruce K. Gale, ${ }^{4}$ Richard K. Watt, ${ }^{1}$ Matthew C. Asplund, ${ }^{*, 1}$ Matthew R. Linford ${ }^{*, 1}$ \\ ${ }^{1}$ Department of Chemistry and Biochemistry, Brigham Young University, Provo, UT 84602, ${ }^{2}$ GE Global Research, 1 \\ Research Circle, Niskavuna, NY, ${ }^{3}$ Department of Chemical Engineering, University of Utah, Salt Lake City, UT \\ 84112, 'Department of Mechanical Engineering, University of Utah, Salt Lake City, UT 84112
}

\section{SUPPORTING INFORMATION}

Experimental Details

Materials Silicon wafers (100), single polished were obtained from Unisil Corporation, Santa Clara, CA. Toluene ( $\geq$ 99.9\%, Sigma, USA), ethanol (100\%, AaperAlcohol, USA), $\mathrm{HCl}$ (concentrated, 37\%, Mallinckrodt Corporate, USA), 2-[methoxy(polyethylenoxy)propyl]trimethoxysilane $\left(\left(\mathrm{CH}_{3} \mathrm{O}\right)_{3} \mathrm{Si}\left(\mathrm{CH}_{2}\right)_{3}\left(\mathrm{OCH}_{2} \mathrm{CH}_{2}\right)_{6-9} \mathrm{OCH}_{3}, \geq 90 \%\right.$, MW 460-590, 6-9 PEG units, Gelest, Tullytown, USA), sodium phosphate monobasic, anhydrous ( $\geq 99 \%$, Sigma, USA), and sodium phosphate dibasic, heptahydrate, $(\geq 99 \%$, Fluka, USA) were used as received. Phosphate buffer $(0.02 \mathrm{M})$ was prepared with sodium phosphate monobasic, anhydrous, and sodium phosphate dibasic, heptahydrate. Albumin from bovine serum (IgG-free, cell culture tested, Sigma, USA), myoglobin (from equine skeletal muscle, 95-100\%, Sigma, USA), lysozyme ( $95 \%$, 50,000 units/mg protein, Sigma, USA), Immunopure Avidin (Pierce Biotechnology, USA), streptavidin (AnaSpec, Inc., USA), Cy3 tagged Protein A (Invitrogen Corporation, USA), biotin-4-fluorescein (Invitrogen Corporation, USA) (the "fluorescein-biotin conjugate"), fluorescein (free acid, Fluka, USA), Protein A (essentially salt-free, Sigma, USA), and ferritin ( from horse spleen, 99.5\%, Sigma) were also used as received. The aqueous buffer ( $\mathrm{pH}$ 7.5) used to dissolve ferritin consisted of $0.05 \mathrm{M} \mathrm{NaCl}$ and $0.05 \mathrm{M}$ MOPS (3-(Nmorpholino)propanesulfonic acid, sodium salt).

PEG Monolayer Preparation Following a literature procedure, ${ }^{1}$ a silane with 6 - 9 polyethylene glycol (PEG) units $\left(\left(\mathrm{CH}_{3} \mathrm{O}\right)_{3} \mathrm{Si}\left(\mathrm{CH}_{2}\right)_{3}\left(\mathrm{OCH}_{2} \mathrm{CH}_{2}\right)_{6-9} \mathrm{OCH}_{3}\right)$ was dissolved in toluene to form a 4-5 mM solution that also contained 0.8 $\mathrm{mL} / \mathrm{L} \mathrm{HCl}$ (conc). Silicon wafers were plasma cleaned for 5 minutes at high power (18W applied to the RF coil) with a basic plasma cleaner (model PDC-32G) from Harrick Plasma (Ithaca, NY), and then immersed into the PEG silane solution for $18 \mathrm{hrs}$ at room temperature. The wafers were then washed with toluene, ethanol, and water, and finally dried with a jet of nitrogen. The resulting films had uniform ellipsometric thicknesses $(15.2 \pm 0.3 \AA)$ and advancing water contact angles $\left(29.8^{\circ} \pm 2.2^{\circ}\right)$. These values are the average of eight measurements from four surfaces.

Surface Patterning with the Microlens Array A microlens array (MLA) with $100 \mu \mathrm{m}$ spacing between microlenses (SUSS MicroOptics, Neuchatel, Switzerland) was placed approximately $200 \mu \mathrm{m}$ over the PEG monolayer coated silicon oxide substrate. A single nanosecond pulse of $15 \mathrm{~mJ} 532 \mathrm{~nm}$ laser light (Coherent Infinity Nd:YAG laser) was then shot through this optical element to pattern the surface. The surface was not cleaned or rinsed in any way after MA patterning.

Protein Adsorption Proteins, except ferritin, were dissolved in phosphate buffered saline (PBS) to form $10^{-5} \mathrm{M}$ solutions. The concentration of ferritin was also $10^{-5} \mathrm{M}$, but it was dissolved in the MOPS buffer described above. A microlens array patterned PEG monolayer on silicon oxide was then immersed into a protein solution overnight at $2-8$ ${ }^{\circ} \mathrm{C}$. The surfaces, including those with ferritin adsorbed on them, were then rinsed with PBS buffer followed by Millipore water, and dried with a jet of $\mathrm{N}_{2}$.

Surface Characterization Planar surfaces described in this work were characterized with spectroscopic ellipsometry (J.A. Woollam M-2000), contact angle goniometry (Rame-Hart model 100-00 contact angle goniometer), X-ray photoelectron spectroscopy (XPS) (Surface Science SSX-100 instrument with a monochromatized Al K $\alpha$ source and a hemispherical analyzer), and time-of-flight secondary ion mass spectrometry (ToF-SIMS) (ION-TOF TOF-SIMS IV instrument with a two-lens ${ }^{69} \mathrm{Ga}^{+}$gun).

Fluorescence System Our imaging system employs a TE 2000-U inverted microscope (Nikon, Tokyo, Japan). The laser light was generated with an air-cooled 35-LAP-321-120 Ar ion laser (Melles Griot, Carlsbad, CA) and directed into the microscope via an optical path consisting of an excitation filter (D488/10, Chroma, Brattleboro, VT), a homemade periscope, a homemade quartz diffuser, which was used to homogenize images, and a light-gathering lens. Fluorescence was collected through a $10 \_0.30$ NA objective (Nikon) and passed through a Z488LP long-pass filter set (Chroma). All images were recorded with a Coolpix 5400 digital camera (Nikon). ${ }^{2}$

Microfluidics Spotting Spotting on MA patterned surfaces was performed with a Continuous Flow Microspotter (CFM) from Wasatch Microfluidics (Salt Lake City, UT). The concentration of the Cy3 tagged Protein A solution was $50 \mu \mathrm{M}$ 


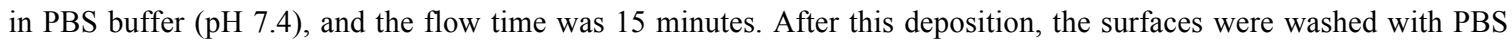
buffer using the spotter. After removal of the spotter the surfaces were washed with water.

Data Analysis Multivariate curve resolution (MCR) of ToF-SIMS images was performed using the AXSIA toolbox that was written at Sandia National Laboratories. Details of data binning and preprocessing have been described previously. ${ }^{3}$ For the data shown here, the mass range of 0 to $1000 \mathrm{amu}$ was binned to $1 \mathrm{amu}$. Retrospective spectral analysis revealed that all of the species of interest were included in this mass range.

Note on Solution $p H$ during Protein Adsorption The solution $\mathrm{pH}$ did not appear to strongly alter protein adsorption, as measured on model, planar, silicon oxide surfaces. For BSA, only a small decrease in protein layer thickness was observed as the $\mathrm{pH}$ of the deposition solution increased: solutions at $\mathrm{pH} 5.6,6.6$, and 7.4 gave ellipsometric thicknesses of $21.4 \pm 0.1 \AA, 18.23 \pm 0.02 \AA$, and $18.6 \pm 0.3 \AA$, respectively. These thickness measurements were consistent with XPS results for these pH values: by XPS the percent nitrogen at the surfaces was 8.6, 7.2, and 6.0. For myoglobin deposited from PBS buffer at pH 5.6 and 8.0, the film thicknesses were $39.5 \pm 0.2 \AA$ and $17.69 \pm 0.03 \AA$, respectively, and the percent nitrogen by XPS was 5.5 and 4.3 , respectively.

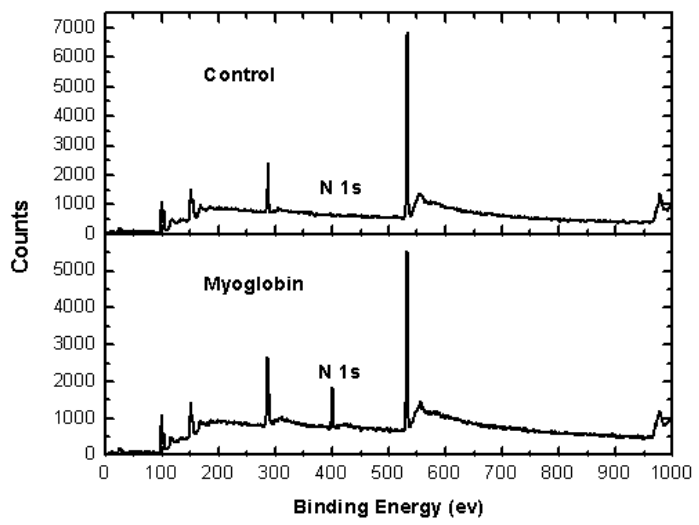

Figure 1, Supporting Information. X-ray photoelectron spectroscopy survey scans of a) an $\mathrm{Si} / \mathrm{SiO}_{2}$ surface and b) a PEG silane monolayer coated $\mathrm{Si} / \mathrm{SiO}_{2}$ surface after immersion in a $\mathrm{pH} 5.6$ solution of myoglobin in PBS buffer.

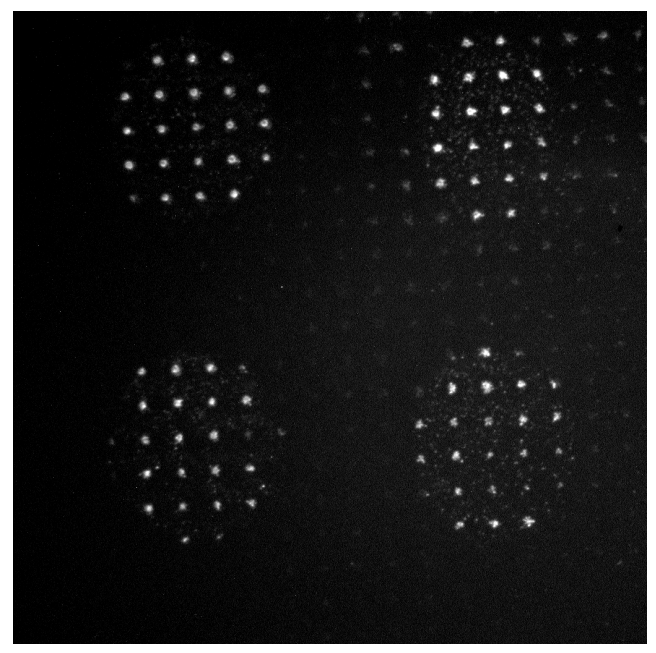

Figure 2, Supporting Information. Fluroescence microscopy image of Cy3 tagged Protein A selectively deposited onto an MA patterned substrate using a continuous flow microspotter. The excitation wavelength was ca. $540 \mathrm{~nm}$ and the emission wavelength was ca. $600 \mathrm{~nm}$. This particular experiment differed from all others reported in this work in that the spacing between the microlenses in the MA was $83 \mu \mathrm{m}$ and the spaces between the microlenses were masked by chromium. 
Table 1. Molecular weights and pl values of the proteins that were studied.

\begin{tabular}{ccc}
\hline Protein & pI & MW (Da) \\
\hline BSA & 4.9 & 66,000 \\
\hline Avidin & 10.5 & 66,000 \\
\hline Streptavidin & 5.5 & 60,000 \\
\hline Lysozyme & 11.35 & 14,700 \\
\hline Myoglobin & 7 & 16,951 \\
\hline Protein A & 6.8 & 42,000 \\
\hline Ferritin & 4.4 & 440,000 \\
\hline
\end{tabular}

Supporting Information References

1. Papra, A.; Gadegaard, N.; Larsen, N.B. Langmuir 2001, 17, 1457-1460.

2. Liu, J.; Sun, X.; Farnsworth, P. B.; Lee, M. L. Anal. Chem 2006, 78, 4654.

3. Ohlhausen, J.A.T.; Keenan, M.R.; Kotula, P.G.; Peebles, D.E. Appl. Surf. Sci. 2004, 231-232, 230-234. 